\title{
Treatment of chronic subdural hematoma with atorvastatin combined with low-dose dexamethasone: phase II randomized proof-of-concept clinical trial
}

\author{
*Dong Wang, MD, PhD, ${ }^{1,2}$ Chuang Gao, MD, PhD, ${ }^{1,2}$ Xin Xu, MD, PhD, , ${ }^{1,2}$ Tao Chen, PhD, ${ }^{3}$ \\ Ye Tian, MD, PhD, , ${ }^{1,2}$ Huijie Wei, MD, ${ }^{1,2}$ Shu Zhang, PhD, ${ }^{2}$ Wei Quan, MD, PhD, ${ }^{1,2}$ Yi Wang, MD, ${ }^{1,2}$ \\ Shuyuan Yue, MD, ${ }^{1,2}$ Zengguang Wang, MD, PhD, ${ }^{1,2}$ Ping Lei, MD, PhD, ${ }^{4}$ Craig Anderson, MD, PhD, ${ }^{5}$ \\ Jingfei Dong, MD, PhD, ${ }^{6}$ Jianning Zhang, MD, PhD, ${ }^{1,2}$ and Rongcai Jiang, MD, PhD ${ }^{1,2}$

\begin{abstract}
'Department of Neurosurgery, Tianjin Medical University General Hospital, Tianjin; ${ }^{2}$ Key Laboratory of Post-Neuroinjury Neuro-repair and Regeneration in the Central Nervous System, Ministry of Education, Tianjin Medical University, Tianjin Key Laboratory of Injury and Regenerative Medicine of Nervous System, Tianjin Neurological Institute, Tianjin, China; ${ }^{3}$ Department of Clinical Sciences, Liverpool School of Tropical Medicine, Liverpool, United Kingdom; ${ }^{4}$ Department of Geriatrics, Tianjin Medical University General Hospital, Tianjin, China; ${ }^{5}$ George Institute China at Peking University Health Science Center China and George Institute for International Health, University of Sydney, Australia; and ${ }^{6}$ Bloodworks Research Institute, Bloodworks Northwest and Department of Medicine, University of Washington School of Medicine, Seattle, Washington
\end{abstract}

OBJECTIVE The authors sought to test the hypothesis that adding dexamethasone (DXM) to atorvastatin (ATO) potentiates the effects of ATO on chronic subdural hematoma (CSDH).

METHODS Sixty patients with CSDH underwent 5 weeks of treatment with an additional 7-week follow-up. Patients were randomized to receive a 5 -week regimen of ATO $20 \mathrm{mg}$ daily or ATO $20 \mathrm{mg}$ daily plus a DXM regimen (ATO+DXM). The 5-week DXM regimen was $2.25 \mathrm{mg}$ daily for 2 consecutive weeks, followed by $0.75 \mathrm{mg}$ twice daily for 2 weeks and $0.75 \mathrm{mg}$ once daily for 1 week. The primary endpoint was hematoma reduction assessed by neuroimaging at baseline and at 5 weeks of follow-up. Secondary outcomes included neurological improvement assessed by using the Markwalder's Grading Scale and Glasgow Coma Scale (MGS-GCS).

RESULTS The mean patient age was 66.6 years, and $25 \%$ of patients were women. The patients who were treated with ATO+DXM had more obvious hematoma reduction at the 5th week (between-groups difference $18.37 \mathrm{ml} ; 95 \% \mathrm{Cl} 8.17$ $28.57 ; p=0.0005)$. This reduction started from the 2 nd week $(14.51 \mathrm{ml} ; 95 \% \mathrm{Cl} 4.31-24.71 ; p=0.0056)$ of treatment and persisted until the 12 th week ( $17.50 \mathrm{ml} ; 95 \% \mathrm{Cl} 7.30-27.70 ; p=0.0009$ ). Complete recovery of neurological function (MGS-GCS grade 0 ) at 5 weeks was achieved in $83.33 \%$ and $32.14 \%$ of patients in the ATO+DXM and ATO groups, respectively. At the 5th week, patients receiving ATO+DXM had significantly lower levels of T cells and higher levels of regulatory $T$ cells and endothelial progenitor cells in their peripheral blood.

CONCLUSIONS ATO+DXM was more effective than ATO alone in reducing hematoma and improving neurological function in patients with CSDH. These results require further confirmation in a randomized placebo-controlled trial.

Clinical trial registration no.: ChiCTR-IPR-14005573 (http://www.chictr.org.cn/index.aspx)

https://thejns.org/doi/abs/10.3171/2019.11.JNS192020

KEYWORDS chronic subdural hematoma; atorvastatin; dexamethasone; endothelial progenitor cells; regulatory T cells; trauma

\footnotetext{
ABBREVIATIONS ADL = activities of daily living; $A D L-B I=A D L$ according to the modified Barthel Index; $A T O=$ atorvastatin; $C S D H=$ chronic subdural hematoma; DXM = dexamethasone; $\mathrm{EPC}=$ endothelial progenitor cell; FACS = fluorescence-activated cell sorting; FITC = fluorescein isothiocyanate; GOSE = Glasgow Outcome ScaleExtended; HV = hematoma volume; LOCF = last observation carried forward; MGS-GCS = Markwalder's Grading Scale and Glasgow Coma Scale; PE = phycoerythrin; Treg = regulatory $T$ cell.
}

SUBMITTED July 25, 2019. ACCEPTED November 18, 2019.

INCLUDE WHEN CITING Published online January 31, 2020; DOI: 10.3171/2019.11.JNS192020.

* D.W., C.G., and X.X. contributed equally to this work. J.Z. and R.J. contributed equally to the supervision of this work. 


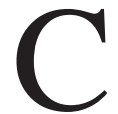
HRONIC subdural hematoma (CSDH) occurs in 1-13.1 per 100,000 persons per year, but its morbidity rate is increasing due to aging of the population and increasing use of anticoagulation/antiplatelet agents. ${ }^{19}$ Twist-drill craniotomy or burr-hole craniostomy to evacuate hematoma remains the first-line treatment for symptomatic patients with $\mathrm{CSDH}$; however, these techniques are accompanied by high rates of recurrence (20\%) and complications. ${ }^{17,20,26}$ Therefore, various drug treatments have been considered, including the use of mannitol, glucocorticoids, angiotensin-converting enzyme inhibitors, tranexamic acid, and platelet activating factor receptor inhibitor, ${ }^{7,11,27}$ but few have been rigorously evaluated in a randomized clinical trial. ${ }^{22}$ High doses of dexamethasone (DXM) have been reported to reduce $\mathrm{CSDH},{ }^{4,10,23}$ but potential benefits of this treatment are offset by complications such as hyperglycemia, infection, gastritis, cellulitis, and cardiac disorders. 5,23,31,35,36 The efficacy of low-dose DXM has not been previously studied.

In 2014, atorvastatin (ATO) was first reported to be safe and effective in prompting the absorption of hematoma based on the results of a preliminary study from 3 medical centers. ${ }^{33}$ Then, in an SDH rat model, ATO was demonstrated to reduce inflammation and hematoma. ${ }^{14}$ In 2017, Chan et al. found that CSDH patients treated with ATO had lower rates of deterioration and burr-hole drainage. ${ }^{6}$ In 2018, Tang et al. analyzed 245 consecutive adult patients undergoing burr-hole craniotomy for CSDH and found that perioperative ATO administration was associated with a lower rate of $\mathrm{CSDH}$ recurrence. ${ }^{30}$ There was also a systematic review which found that oral ATO may be beneficial in the management of $\mathrm{CSDH} .{ }^{24}$ In a recent double-blind, randomized, placebo-controlled clinical trial, we showed that $20 \mathrm{mg}$ ATO given daily for 8 weeks is safe and effective in reducing hematoma and improving the clinical outcomes of patients with CSDH. ${ }^{12}$ This trial was developed based on previous studies, including our own, showing that statins have an antiinflammatory effect and can mobilize endothelial progenitor cells (EPCs) for vascular repair. ${ }^{1,15,21}$ However, as approximately $11.2 \%$ of participants in our trial failed to respond to ATO treatment and more prolonged treatment could increase the risk of drug-related complications ${ }^{3}$ and noncompliance, we hypothesized that the clinical efficacy of ATO can be enhanced or accelerated when ATO is combined with a low-dose regimen of DXM. Such an approach might avoid complications associated with high-dose DXM. Herein, we report the results from a phase II clinical trial designed as an initial test of this hypothesis.

\section{Methods}

This open-label, evaluator-blinded, parallel, proof-ofconcept, randomized trial was conducted at the General Hospital of Tianjin Medical University, Tianjin, China, between July 2014 and December 2018.

\section{Patient Recruitment}

The patients who met the inclusion criteria were adult (age $\geq 18$ years) men and nonpregnant women with a diagnosis of primary supratentorial CSDH with minimal mid- line shift $(<1 \mathrm{~cm})$ confirmed on brain CT or MRI who were determined by 2 attending surgeons to be at no risk of cerebral hernia and not in need of immediate surgery. In addition, participants had minimal loss of consciousness (Markwalder's Grading Scale and Glasgow Coma Scale [MGS-GCS] ${ }^{18,29}$ grade $<3$, Supplementary Table e-1) and no previous surgery for CSDH. These inclusion criteria were similar to those for our previously reported ATOCH (Effect of Atorvastatin on Chronic Subdural Hematoma) trial. ${ }^{12}$ Patients were excluded if they were allergic to statins; had a secondary cause of the hematoma (e.g., tumor or bleeding diathesis), severe comorbidity, or liver disease; had received a statin or steroid within the last week or were required to take statins in the next 3 months; had diabetes mellitus with poorly controlled blood glucose; had uncontrolled hypertension or infection; or had already participated in clinical trials in the previous 4 weeks. After patient recruitment, a random number sequence was generated with computer software by a statistician who was not involved in the study team, and patients were allocated to either the ATO group or the ATO+DXM group according to the random number. Since this was a proof-of-concept study, no formal calculation was performed to determine the sample size. ${ }^{8}$ However, based on a planned study period of 2014-2018, we estimated that 60 patients would be recruited into the 2-arm trial.

\section{Standard Protocol Approvals, Registrations, and Patient Consent}

The trial protocols and documentation were approved by the ethics committee of the Tianjin Medical University General Hospital Institutional Review Board, and written informed consent was obtained from each participant or his/her legal surrogate. The trial was registered on the website of the Chinese Clinical Trial Registry (http:// www.chictr.org.cn/index.aspx) with the registration number ChiCTR-IPR-14005573. There were no important changes to the methods after trial commencement and no interim analyses were performed.

\section{Treatment and Follow-Up}

Sixty eligible patients were randomly assigned in a 1:1 ratio to one of the two treatment regimens: patients in the ATO group received $20 \mathrm{mg}$ of oral ATO (Pfizer Inc.) once daily for 5 consecutive weeks and those in the ATO-DXM group received $20 \mathrm{mg}$ of ATO and DXM (Tianyao Pharmaceutical Co. Ltd.) daily for 5 consecutive weeks. DXM was administered in the following doses: $2.25 \mathrm{mg}$ daily for 2 weeks followed by $0.75 \mathrm{mg}$ twice daily for 2 weeks and subsequently at $0.75 \mathrm{mg}$ once a day for 1 week. All patients were then followed up for an additional 7 weeks after cessation of the drug treatment. Treatment compliance was monitored by regular contact with participants and their caregivers through telephone and during weekly visits for clinical evaluation and instructions on DXM dosage. Patients could be switched to surgery to evacuate the hematoma when their neurological dysfunction significantly deteriorated (worsened headache, progressive limb paralysis, or changes in the levels of consciousness) 


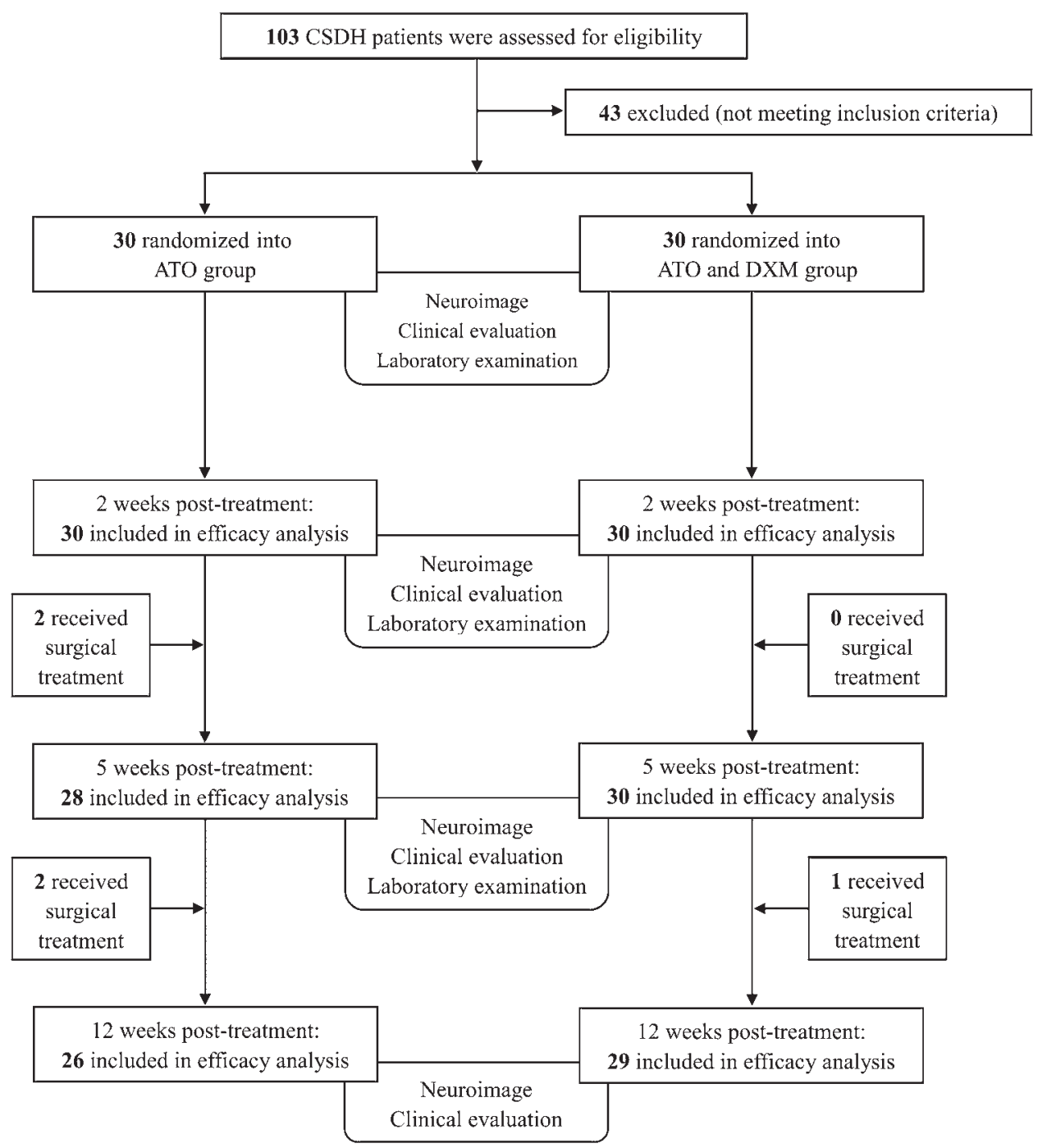

FIG. 1. Schematic illustration of the trial protocol.

or when CT or MRI scans showed hematoma enlargement and/or a midline shift $(>1 \mathrm{~cm})$ during follow-up.

\section{Outcome Assessment}

The primary endpoint was hematoma reduction between baseline and 5 weeks after the first dose, based on assessment of the CT and MR images by 3 neuroradiologists blinded to treatment allocation and other details. Hematoma volume (HV) was calculated using the Tada formula: maximal length $\times$ maximal width $\times$ maximal thickness of the hematoma, divided by $2 .{ }^{13}$ The HV was calculated bilaterally and the total $\mathrm{HV}$ was calculated as the HV of the left plus the HV of the right.

Secondary outcomes included MGS-GCS score at the time of the first drug dose and at the end of weeks 5 and 12 , evaluated by 2 attending neurosurgeons blinded to treatment; reduction in $\mathrm{HV}$ at the end of weeks 2 and 12 after the first dose (during the follow-up period); neurological function assessed by the ability of a patient to perform activities of daily living (ADL) according to the modified
Barthel Index (ADL-BI) and the patient's Glasgow Outcome Scale-Extended (GOSE) score at the end of weeks 5 and 12 after the first dose; rate of surgical intervention; and laboratory results.

\section{Fluorescence-Activated Cell Sorting Assessment}

Of the 30 patients in each group, 24 patients in the ATO group and 26 patients in the ATO+DXM group had undergone blood collection on admission. Fluorescence-activated cell sorting (FACS) assessment was performed in all of these patients before the treatment as well as 2 and 5 weeks after the treatment. Peripheral blood samples were collected from a subgroup of patients using $0.32 \%$ sodium citrate as an anticoagulant (final concentration) for flow cytometry (FACS Aria III; BD Biosciences) analysis of EPCs, regulatory $\mathrm{T}$ cells (Tregs), and $\mathrm{T}$ lymphocyte subtypes at baseline (before treatment) and at weeks 2 and 5 . These measurements were surrogate markers of systemic inflammation or vascular repair. To measure EPCs, mononuclear cells were isolated from whole blood by density 
gradient centrifugation at $300 \mathrm{~g}$ for 20 minutes at room temperature and stained with fluorescein isothiocyanate (FITC)-CD34 and phycoerythrin (PE)-CD133 antibodies (Miltenyi Biotec). ${ }^{16}$ Tregs were detected by using a commercial kit (130-094-158; Miltenyi Biotec), according to the manufacturer's instructions. T-lymphocyte subtypes were identified by FITC-CD4, PE-CD8, and peridininchlorophyll-protein complex (PerCP)-CD19 antibodies, as previously described. ${ }^{8}$ The flow cytometry data were analyzed using FlowJo software (Tree Star).

\section{Laboratory Assays}

Blood was analyzed for complete blood cell count, international normalized ratio, activated partial thromboplastin time, plasma fibrinogen and D-dimer, serum alanine aminotransferase, aspartate transaminase, gamma glutamyl transpeptidase, urea nitrogen, creatinine, and blood glucose at baseline and at weeks 2 and 5 .

\section{Safety Monitoring}

We recorded all adverse events identified when patients were in the hospital or at outpatient clinic visits during follow-up. A serious adverse clinical event was defined as a significant hazard or adverse effects that were related to the trial drugs and required treatment. As ATO and DXM are associated with abnormal glucose metabolism, hyperglycemia and hyperglycemia-related nosocomial infections were also monitored.

\section{Statistical Analysis}

Using the intention-to-treat principle, we performed an efficacy analysis that included all the patients randomized (full analysis set). Adverse events were assessed in all patients who received at least one dose of a treatment drug before the end of follow-up.

We present continuous variables as means \pm standard deviations and categorical variables as frequencies and percentages. Differences between treatment groups were assessed using the independent-samples t-test for continuous measures and the chi-square test for categorical variables (or Fisher's exact test when the expected value was $<5$ ).

For the primary endpoint, we used a linear mixed-effects model to estimate the difference in hematoma reduction. The model was fitted with treatment, time, interaction between treatment and time, baseline HV, age, and sex as fixed effects and the patient as the random effect. The last observation carried forward (LOCF) method was used to fill in missing data.

Correlations between HVs and cellular markers on FACS were determined using Pearson's coefficient. All analyses were performed using SPSS statistical software (version 22.0; IBM Corp.) and SAS (version 9.4; SAS Institute), and the significance threshold was set at $\mathrm{p}<0.05$ (2-tailed).

\section{Results}

A flowchart of patient recruitment to this study is shown in Fig. 1. During July 2014 and December 2018, 315 CSDH
TABLE 1. Baseline demographic and clinical characteristics of patients randomized to the ATO or ATO+DXM group

\begin{tabular}{|c|c|c|c|}
\hline & ATO $(n=30)$ & $\begin{array}{c}\text { ATO+DXM } \\
(\mathrm{n}=30)\end{array}$ & $\begin{array}{c}p \\
\text { Value }\end{array}$ \\
\hline Age, yrs & $63.83 \pm 13.73$ & $69.37 \pm 10.9$ & $0.089^{*}$ \\
\hline Sex & & & $0.136 \dagger$ \\
\hline Male & 20 & 25 & \\
\hline Female & 10 & 5 & \\
\hline CSDH w/ TBI history & $21(70 \%)$ & $19(63.33 \%)$ & $0.584 \dagger$ \\
\hline MGS-GCS score & & & $0.687 \dagger$ \\
\hline 0 & 3 & 4 & \\
\hline 1 & 23 & 20 & \\
\hline 2 & 4 & 6 & \\
\hline ADL-BI score & & & $0.766 \dagger$ \\
\hline$\geq 85$ & 22 & 23 & \\
\hline$<85$ & 8 & 7 & \\
\hline Baseline hematoma vol, ml & $60.77 \pm 27.62$ & $67.48 \pm 21.22$ & $0.296^{*}$ \\
\hline Hematoma location & & & $0.160 \dagger$ \\
\hline Right & 14 & 7 & \\
\hline Left & 9 & 14 & \\
\hline Bilateral & 7 & 9 & \\
\hline \multicolumn{4}{|l|}{ Medical history } \\
\hline Hypertension & $7(23.33 \%)$ & $10(33.33 \%)$ & $0.39 \dagger$ \\
\hline Diabetes & $5(16.67 \%)$ & $5(16.67 \%)$ & $1.000 \dagger$ \\
\hline Cardiac disease & $6(20 \%)$ & $9(30 \%)$ & $0.371 \dagger$ \\
\hline \multicolumn{4}{|l|}{ Medication history } \\
\hline Antiplatelet therapy & $3(10 \%)$ & $2(6.67 \%)$ & $1.000 \ddagger$ \\
\hline Anticoagulant therapy & $0(0 \%)$ & $1(3.33 \%)$ & $1.000 \ddagger$ \\
\hline \multicolumn{4}{|l|}{ Social history } \\
\hline Smoker & $10(33.33 \%)$ & $9(30 \%)$ & $0.781 \dagger$ \\
\hline Drinker & $7(23.33 \%)$ & $9(30 \%)$ & $0.559 \dagger$ \\
\hline
\end{tabular}

Values are presented as number of patients (\%) unless otherwise indicated.

Mean values are presented \pm SD.

* Independent-samples t-test.

† Chi-square test.

$\ddagger$ Fisher's exact test.

patients were admitted to our department. In total, 212 of these patients were treated with surgery directly, and 103 patients were screened for eligibility of this trial. Of these 103 patients, 43 patients were excluded because they did not meet the inclusion criteria. Baseline demographic and clinical characteristics were comparable between the $2 \mathrm{pa}-$ tient groups (Table 1 and Supplementary Tables e-2-1 and e-2-2). Among the 103 patients screened, 60 were enrolled (mean age $66.6 \pm 12.6$ years). There were no deaths or dropouts during the trial, but 3 and 2 patients in the ATO and ATO+DXM groups, respectively, did not undergo neuroimaging at weeks 2 and 5. Table 1 shows that baseline HVs were comparable between the 2 groups $(60.77 \pm 27.62$ vs $67.48 \pm 21.22 \mathrm{ml} ; \mathrm{p}=0.296$ ). Consistent with findings of our recent trial, patients receiving ATO alone had a 24.3\% reduction in $\mathrm{HV}$ at the end of week 5. However, patients in the ATO+DXM group had a significantly greater reduc- 

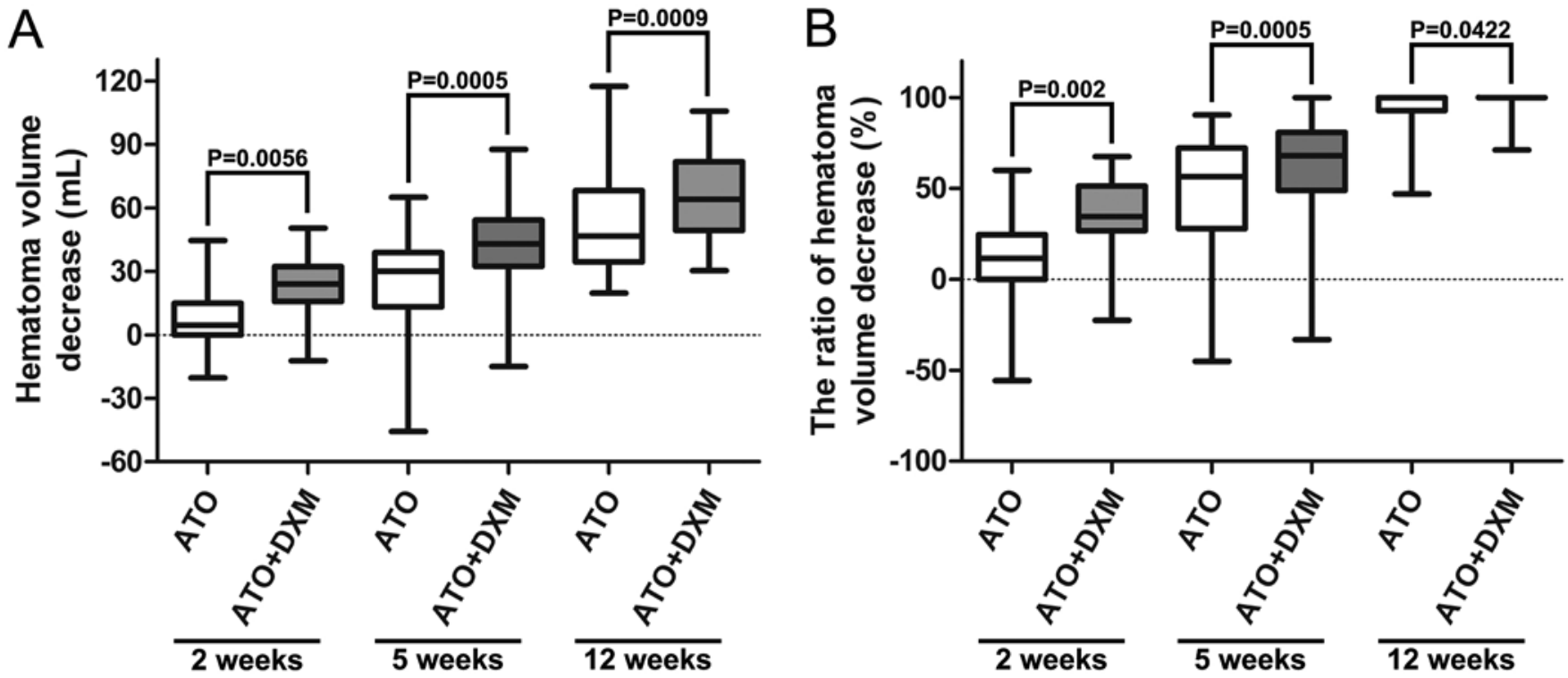

FIG. 2. Reduction in HV after 2, 5, and 12 weeks of treatments. A: The absolute hematoma reduction was calculated as HV at baseline - HV after treatment. Patients treated with ATO+DXM had greater hematoma reduction than those treated with ATO alone after week 2 (7.02 \pm 13.06 vs $23.11 \pm 15.37 \mathrm{ml}$, mean difference $14.51 \mathrm{ml} ; 95 \% \mathrm{Cl} 4.31-24.71 ; p=0.0056)$, week $5(23.32 \pm$ 26.35 vs $43.27 \pm 19.09 \mathrm{ml} ; p=0.0005)$, and week 12 (44.71 \pm 35.92 vs $63.78 \pm 25.45 \mathrm{ml} ; p=0.0009)$ of treatment. B: The relative hematoma reduction was calculated as (HV at baseline $-\mathrm{HV}$ after treatment)/HV at baseline $\times 100 \%$. The ATO+DXM group had an increased percentage of hematoma reduction at week $2(34.47 \% \pm 22.17 \%$ vs $12.16 \% \pm 22.08 \%, p=0.002)$, week $5(64.80 \%$ $\pm 26.46 \%$ vs $39.46 \% \pm 41.61 \%, p=0.0005)$, and week $12(92.52 \% \pm 24.75 \%$ vs $78.50 \% \pm 43.59 \%, p=0.0422)$. The data are presented as mean \pm standard deviation and were analyzed using a linear mixed model with treatment, time, interaction between treatment and time, baseline HV, age, and sex as fixed effects and the patient as the random effect. The missing data were filled in by using the LOCF method.

tion in HV than those in the ATO group at weeks 2 (7.02 \pm 13.06 vs $23.11 \pm 15.37 \mathrm{ml} ; \mathrm{p}=0.0056), 5(23.32 \pm 26.35$ vs $43.27 \pm 19.09 \mathrm{ml} ; \mathrm{p}=0.0005)$, and $12(44.71 \pm 35.92$ vs $63.78 \pm 25.45 \mathrm{ml} ; \mathrm{p}=0.0009)$ (Fig. 2A). Since the starting $\mathrm{HV}$ varied considerably, the relative hematoma reduction, measured as ( $\mathrm{HV}$ at baseline - $\mathrm{HV}$ after treatment)/ $\mathrm{HV}$ at baseline $\times 100 \%$, was then compared between the 2 groups at different time points. The ATO+DXM group had an increased percentage of hematoma reduction compared with the ATO group at weeks $2(34.47 \% \pm 22.17 \%$ vs $12.16 \% \pm 22.08 \%, \mathrm{p}=0.002), 5(64.80 \% \pm 26.46 \%$ vs $39.46 \% \pm 41.61 \%, \mathrm{p}=0.0005)$, and $12(92.52 \% \pm 24.75 \%$ vs $78.50 \% \pm 43.59 \%, p=0.0422$ ) (Fig. 2B). Patients in the ATO+DXM group also showed remarkably greater improvement in MGS-GCS scores than patients in the ATO group after 5 weeks of treatment $(p=0.0004)$ but not after 2 weeks of treatment (Table 2). Overall, $83.33 \%$ of patients treated with ATO+DXM and $32.14 \%$ of those treated with ATO alone achieved complete recovery of neurological dysfunction (MGS-GCS score 0 ) by week 5 .

Figure 3 shows typical imaging findings in patients with CSDH treated with ATO and ATO+DXM, and Supplementary Fig. e-1 shows changes in imaging findings in the ATO and ATO+DXM groups. There were no statistically significant differences in the MGS-GCS scores between the ATO and ATO+DXM groups after 12 weeks of treatment (Table 2). The frequency of ADL-BI scores $\geq 85$ or $=100$ was comparable between the 2 groups at weeks 5 and $12(\mathrm{ADL}-\mathrm{BI} \geq 85, \mathrm{p}=0.187$ and $0.473 ; \mathrm{ADL}-\mathrm{BI}=100$, $\mathrm{p}=0.737$ and 1.000 , respectively). GOSE scores showed a trend toward improvement in patients receiving combined therapy at weeks 5 and 12 (GOSE $\geq 7, p=0.415$ and 0.473 ; GOSE $=8, p=0.242$ and 0.696 , respectively).

Four patients from the ATO group and 1 from the ATO+DXM group $(13.3 \%$ vs $3.3 \%, p=0.353)$ were switched to surgery at $14,16,40,48$, or 36 days after the first dose due to increasing HVs and exacerbated neurological dysfunction, which was shown as an increased MGSGCS score (Supplementary Tables e-2-1 and e-2-2). Two of the 4 patients in the ATO group were switched to surgery after 2 weeks of treatment and the other 2 patients after 5 weeks. In the ATO+DXM group, 1 patient underwent surgical hematoma drainage on day 48 after the first dose.

The baseline characteristics of the patients who received the FACS assessment showed no significant differences (Supplementary Table e-4). The numbers of circulating EPCs were similar between the 2 groups of patients at baseline but were significantly elevated in patients receiving ATO+DXM at week 2; however, the increase was no longer detectable at 5 weeks of treatment (Fig. 4B). The levels of circulating Treg cells were higher in patients receiving ATO+DXM at weeks 2 and 5 (Fig. 4C). Conversely, CD4 ${ }^{+} \mathrm{T}$ lymphocytes decreased from the baseline values in patients receiving ATO-DXM compared to those receiving ATO alone (Fig. 4D). Numbers of circulating $\mathrm{CD}^{+} \mathrm{T}$ cells and $\mathrm{CD} 19^{+} \mathrm{B}$ cells were similar between the 2 groups of patients (Fig. 4E and F).

One patient who was a carrier of hepatitis B virus at 
TABLE 2. Clinical assessment of patients randomized to the ATO or ATO+DXM group

\begin{tabular}{|c|c|c|c|}
\hline Scale & ATO $(n=30)^{*}$ & ATO+DXM $(n=30)^{*}$ & $\mathrm{p}$ Value \\
\hline MGS-GCS score & & & $0.687 \dagger$ \\
\hline \multicolumn{4}{|l|}{ Baseline } \\
\hline 0 & 3 & 4 & \\
\hline 1 & 23 & 20 & \\
\hline 2 & 4 & 6 & \\
\hline 2 wks & & & $0.435 \dagger$ \\
\hline 0 & 7 & 11 & \\
\hline 1 & 19 & 17 & \\
\hline 2 & 4 & 2 & \\
\hline 5 wks & & & $0.0004 \dagger$ \\
\hline 0 & $9^{a}$ & 25 & \\
\hline 1 & $17^{a}$ & 4 & \\
\hline 2 & $2^{a}$ & 1 & \\
\hline 12 wks & & & $0.286 \dagger$ \\
\hline 0 & $25^{b}$ & $29^{c}$ & \\
\hline 1 & $1^{\mathrm{b}}$ & $0^{c}$ & \\
\hline 2 & $0^{\mathrm{b}}$ & $0^{c}$ & \\
\hline \multicolumn{4}{|l|}{ ADL-BI score } \\
\hline \multicolumn{4}{|l|}{$\geq 85$} \\
\hline Baseline & 22 & 23 & $0.766 \dagger$ \\
\hline 5 wks & $24^{a}$ & 29 & $0.187 \ddagger$ \\
\hline 12 wks & $25^{b}$ & $29^{c}$ & \\
\hline \multicolumn{4}{|l|}{$<85$} \\
\hline Baseline & 8 & 7 & $0.766 \dagger$ \\
\hline 5 wks & $4^{a}$ & 1 & $0.187 \ddagger$ \\
\hline 12 wks & $1^{\mathrm{b}}$ & $0^{c}$ & $0.473 \ddagger$ \\
\hline \multicolumn{4}{|l|}{ ADL-BI 100} \\
\hline Baseline & $17(56.67 \%)$ & $10(33.33 \%)$ & $0.069 \dagger$ \\
\hline 5 wks & $18(64.29 \%)^{a}$ & $18(60 \%)$ & $0.737 \dagger$ \\
\hline 12 wks & $22(84.61 \%)^{b}$ & $25(86.21 \%)^{c}$ & $1.000 \ddagger$ \\
\hline \multicolumn{4}{|l|}{ GOSE score } \\
\hline \multicolumn{4}{|l|}{$\geq 7$} \\
\hline 5 wks & $24^{a}$ & 28 & $0.415 \ddagger$ \\
\hline 12 wks & $25^{b}$ & 2 & $0.473 \ddagger$ \\
\hline \multicolumn{4}{|l|}{$<7$} \\
\hline 5 wks & $4^{a}$ & 29 & $0.415 \ddagger$ \\
\hline 12 wks & $1^{\mathrm{b}}$ & $0^{c}$ & $0.473 \ddagger$ \\
\hline \multicolumn{4}{|l|}{8} \\
\hline 5 wks & $8(28.57 \%)^{a}$ & $13(43.33 \%)$ & \\
\hline 12 wks & $22(84.62 \%)^{b}$ & $26(89.66 \%)^{c}$ & \\
\hline
\end{tabular}

Values are presented as number of patients (\%) unless otherwise indicated.

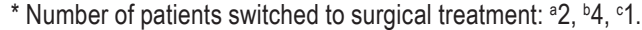

$\dagger$ Chi-square test.

$\ddagger$ Fisher's exact test.

enrollment had elevated aminotransferase levels during the clinical trial, which returned to the baseline after 2 weeks of treatment with bicyclol and glutathione. Four patients had mild liver abnormalities and 1 patient had mild elevation of creatinine, which did not require treatment. Known steroid-related complications were also found, such as mild glycemia in 3 patients with a prior diagnosis of diabetes mellitus, nonspecific nausea in 2 patients, and weight gain in 8 patients. There were no cases of infection, gastrointestinal bleeding, muscle soreness, or rhabdomyolysis (Supplementary Table e-3). Moreover, there were no statistical differences in laboratory results between the 2 groups (Supplementary Table e-3).

\section{Discussion}

Our clinical trial showed that in patients with moderate-sized CSDH, the combined treatment with low-dose ATO and DXM resulted in quicker reduction in HVs, more rapid recovery of neurological function, and a reduced rate of surgery compared to treatment with ATO alone. The treatments were well tolerated during the 12-week trial. These results provide promise that short-term low-dose combination treatment might be effective for patients with mild to moderate forms of CSDH.

To explore the mechanisms underlying the accelerated hematoma reduction with combined treatment, several plasma markers of vascular repair (EPCs) and inflammation (T cells) were serially measured in a subgroup of patients. Changes in EPC and lymphocyte subpopulations in association with reduction in HVs during the course of treatment are consistent with findings of our previous reports that ATO possesses antiinflammatory activity and promotes angiogenesis..$^{14,25,34}$ Our results provide further support for the synergistic action of low-dose DXM with ATO, as demonstrated by downregulation of $\mathrm{CD}^{+}$cells and upregulation of $\mathrm{CD} 4{ }^{+}$Tregs, which are related to cellular immunity. ${ }^{9}$

Combination treatment also appears to increase the levels of circulating EPCs, which are important for vascular repair. ${ }^{2}$ We have previously shown that patients with CSDH have low levels of circulating EPCs, a condition that is associated with hematoma development and recurrence. ${ }^{28}$ ATO increases the levels of EPCs and promotes angiogenesis in mouse models. ${ }^{32,34}$ This study's findings suggest an additive effect in increasing EPCs when using DXM in combination with ATO compared with ATO alone.

We recognize that our study is limited to being a hypothesis-generating proof-of-concept study and lacks sufficient power for reliable assessment of the treatment's effects on clinical outcomes and subgroup analysis. Bias may also have been introduced from the open-label design, but there were no differences in patient background and standard management, and the imaging and clinical outcomes were assessed by physicians blinded to treatment allocation.

\section{Study Limitations}

Our previous studies demonstrated the beneficial effects of ATO therapy for CSDH patients. Therefore, treating CSDH patients without using medical therapy did not meet our ethical principles. This resulted in the lack of a control group with no medical treatment in this study, which was the major limitation of this study. 

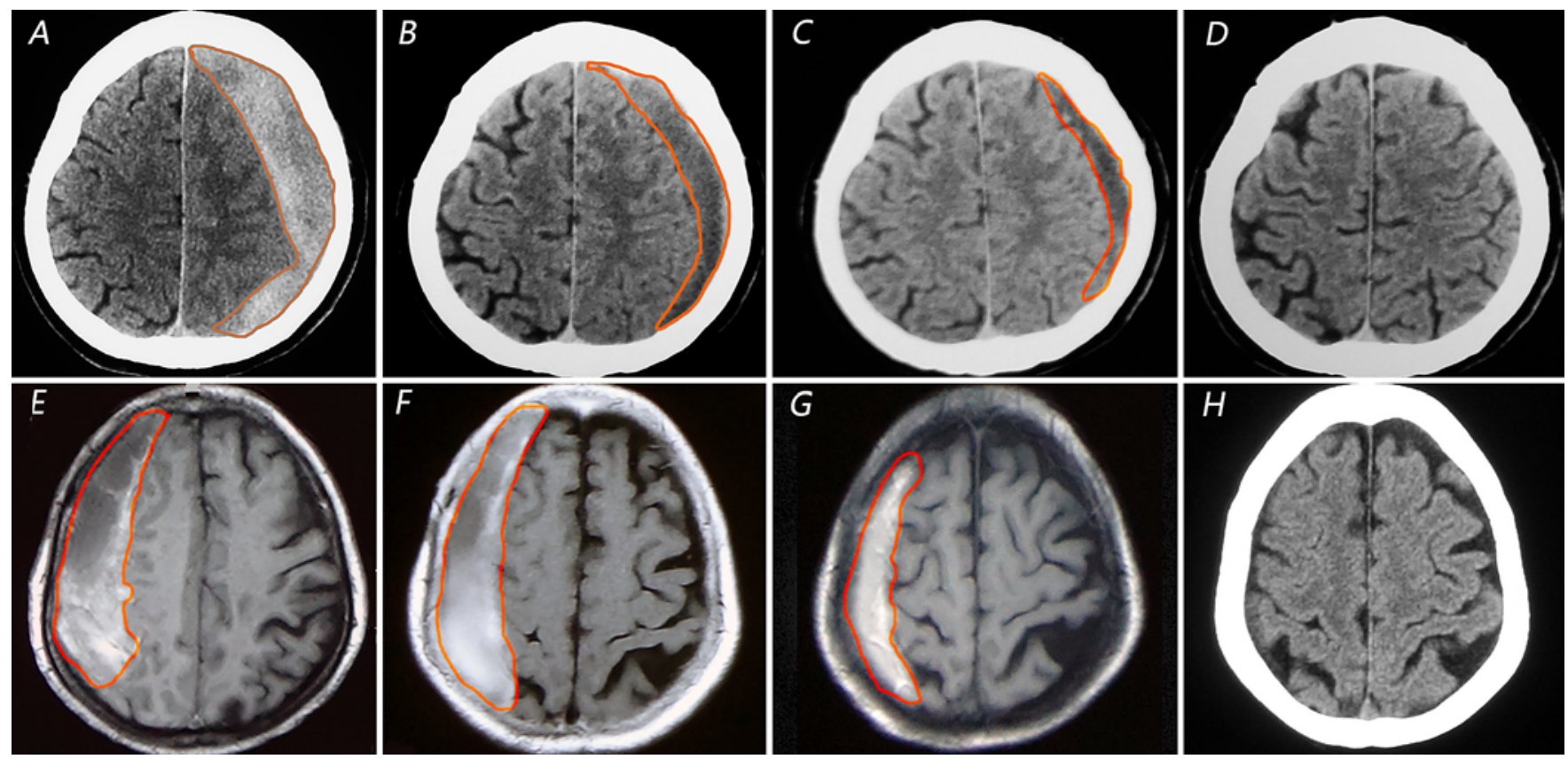

FIG. 3. Representative CT and MR images of CSDH patients treated with ATO or ATO+DXM. A-D: A typical case in the

ATO+DXM group (patient 14), showing rapid absorption of hematoma. The HV was $90.10 \mathrm{ml}$ at baseline (A), $40.54 \mathrm{ml}$ at week 2

(B), $17.84 \mathrm{ml}$ at week $5(\mathrm{C})$, and $0 \mathrm{ml}$ at week 12 (D). E-H: A typical case in the ATO group (patient 5), showing dynamic changes

in HV. The volume was $95.33 \mathrm{ml}$ at baseline $(\mathbf{E}), 50.75 \mathrm{ml}$ at week $2(\mathbf{F})$, and $30.28 \mathrm{ml}$ at week $5(\mathbf{G})$, and the hematoma had disap-

peared by week $12(\mathbf{H})$. Figure is available in color online only.
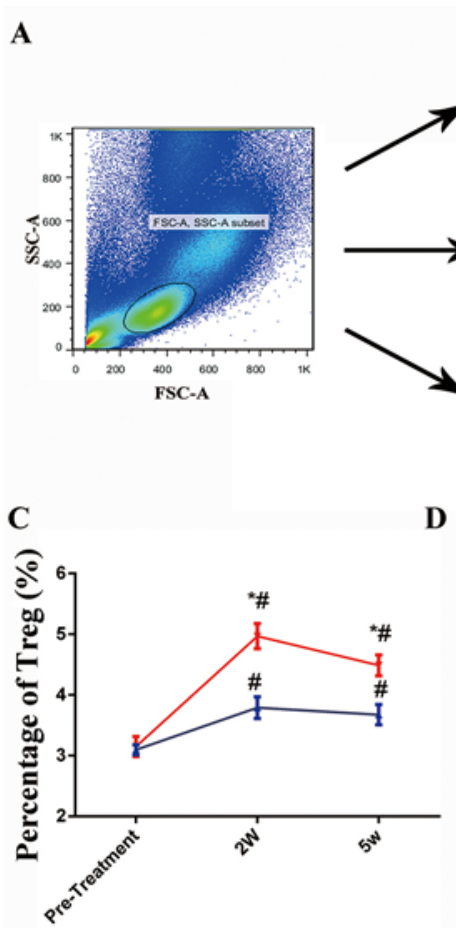

D
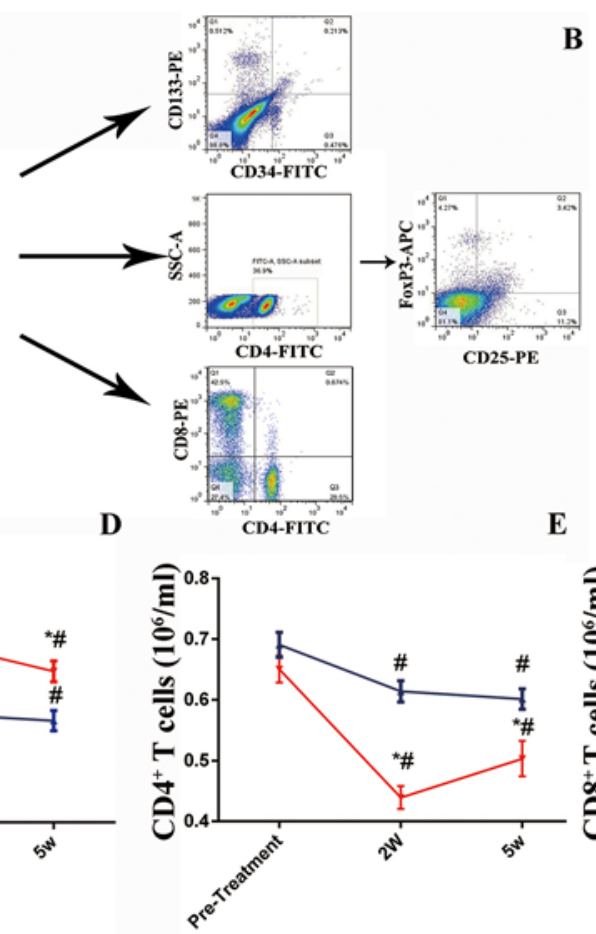

B

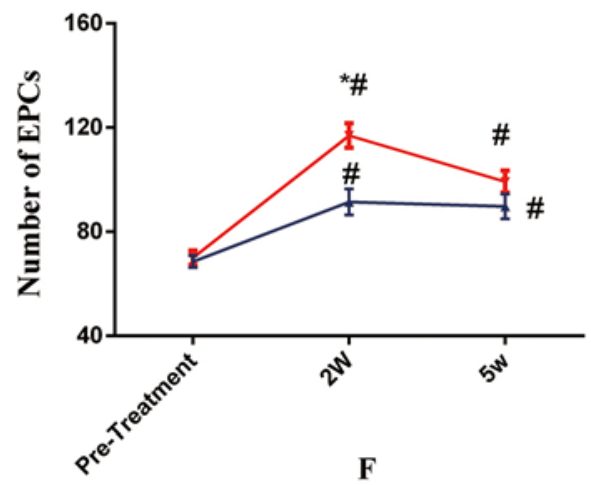

- ATO

$\rightarrow$ ATO+DXM

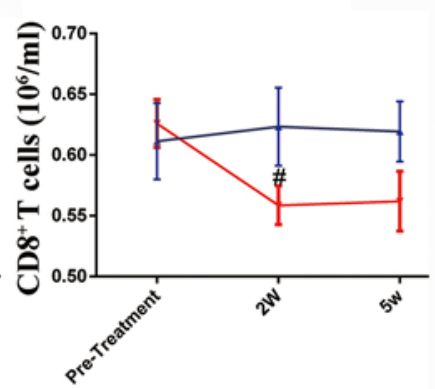

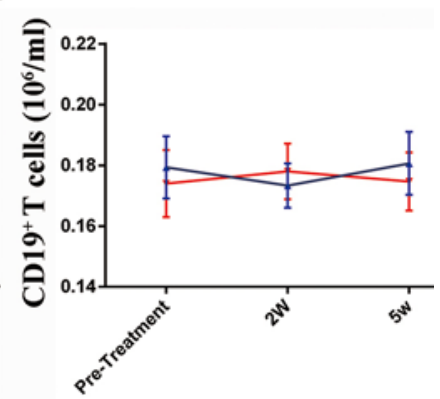

FIG. 4. FACS analysis of circulating EPCs and lymphocyte subtypes. A: Gating strategy for EPCs and lymphocyte subtypes.

$B:$ The number of EPCs increased after both the ATO and ATO+DXM treatments (\#p <0.05). EPCs were significantly increased in the ATO+DXM group compared with the ATO group at 2 weeks ( $\left.{ }^{*} p<0.05\right)$, but showed no difference at 5 weeks. C: The percentage of Tregs among the CD4 ${ }^{+}$T cells was higher in the ATO+DXM group than in the ATO group at 2 and 5 weeks $\left({ }^{*} p<0.05\right)$.

D: The counts of $C D 4^{+}$cells were significantly lower in the ATO+DXM group than in the ATO group at 2 and 5 weeks ( $\left.{ }^{*} p<0.05\right)$.

$\mathrm{E}$ and F: There were no differences in the CD8 ${ }^{+}$T-cell counts and CD19+ B-cell counts between the 2 groups ( $\left.{ }^{*} \mathrm{p}>0.05\right)$.

${ }^{*}=$ compared with the ATO group at the same time point; \# = compared with pretreatment in the same group; FSC = forward scat-

ter; SSC = side scatter; $\mathrm{W}=$ weeks. Figure is available in color online only. 


\section{Conclusions}

In summary, in this open-label, evaluator-blinded trial we found that compared with ATO alone, the combination of ATO and low-dose DXM accelerates hematoma reduction and neurological improvement in patients with CSDH. These results support the existence of potent synergistic effects between the 2 drugs, which act on immunoregulation and vascular repair. The positive proof-of-concept results provide justification to proceed with a formative phase III clinical trial to reliably assess the effects of this combination treatment in CSDH.

\section{Acknowledgments}

This study was funded by the National Natural Science Foundation of China (81671380 to D.W.; 81671221 to R.J.); the Tianjin Research Program of Application Foundation and Advanced Technology (17JCZDJC35900 to D.W.; 14ZCZDSY00179 to R.J.; 18ZLZXZF00270 to Z.W.); the Key Program for International S\&T Cooperation Project of China (81720108015 to J.Z.); the Municipal Science and Technology Commission (15ZXLCSY00060 to J.Z.; 15ZXLCSY00040 to J.Z.); the Science \& Technology Development Fund of the Tianjin Education Commission for Higher Education (2016YD02 to Y.W.); the Scientific Research Program Project of the Tianjin Education Commission (2018ZD03 to Z.W.); the Tianjin Science and Technology Projects in Key Areas of Traditional Chinese Medicine (2018001 to Z.W.); and the Clinical Study of Tianjin Medical University (2017kylc007 to R.J).

\section{References}

1. Araújo FA, Rocha MA, Mendes JB, Andrade SP: Atorvastatin inhibits inflammatory angiogenesis in mice through down regulation of VEGF, TNF-alpha and TGF-beta1. Biomed Pharmacother 64:29-34, 2010

2. Asahara T, Murohara T, Sullivan A, Silver M, van der Zee R, $\mathrm{Li} \mathrm{T}$, et al: Isolation of putative progenitor endothelial cells for angiogenesis. Science 275:964-967, 1997

3. Bakker-Arkema RG, Davidson MH, Goldstein RJ, Davignon $\mathrm{J}$, Isaacsohn JL, Weiss SR, et al: Efficacy and safety of a new HMG-CoA reductase inhibitor, atorvastatin, in patients with hypertriglyceridemia. JAMA 275:128-133, 1996

4. Bender MB, Christoff N: Nonsurgical treatment of subdural hematomas. Arch Neurol 31:73-79, 1974

5. Berghauser Pont LM, Dammers R, Schouten JW, Lingsma HF, Dirven CM: Clinical factors associated with outcome in chronic subdural hematoma: a retrospective cohort study of patients on preoperative corticosteroid therapy. Neurosurgery 70:873-880, 2012

6. Chan DY, Chan DT, Sun TF, Ng SC, Wong GK, Poon WS: The use of atorvastatin for chronic subdural haematoma: a retrospective cohort comparison study. Br J Neurosurg 31:72-77, 2017

7. Edlmann E, Giorgi-Coll S, Whitfield PC, Carpenter KLH, Hutchinson PJ: Pathophysiology of chronic subdural haematoma: inflammation, angiogenesis and implications for pharmacotherapy. J Neuroinflammation 14:108, 2017

8. Fu Y, Hao J, Zhang N, Ren L, Sun N, Li YJ, et al: Fingolimod for the treatment of intracerebral hemorrhage: a 2-arm proofof-concept study. JAMA Neurol 71:1092-1101, 2014

9. Gerriets VA, Kishton RJ, Johnson MO, Cohen S, Siska PJ, Nichols AG, et al: Foxp3 and Toll-like receptor signaling balance Treg cell anabolic metabolism for suppression. Nat Immunol 17:1459-1466, 2016

10. Giamarellos-Bourboulis EJ, Dimopoulou I, Kotanidou A, Livaditi O, Pelekanou A, Tsagarakis S, et al: Ex-vivo effect of dexamethasone on cytokine production from whole blood of septic patients: correlation with disease severity. Cytokine 49:89-94, 2010

11. Holl DC, Volovici V, Dirven CMF, Peul WC, van Kooten F, Jellema K, et al: Pathophysiology and nonsurgical treatment of chronic subdural hematoma: from past to present to future. World Neurosurg 116:402-411.e2, 2018

12. Jiang R, Zhao S, Wang R, Feng H, Zhang J, Li X, et al: Safety and efficacy of atorvastatin for chronic subdural hematoma in Chinese patients: a randomized clinical trial. JAMA Neurol 75:1338-1346, 2018

13. Kothari RU, Brott T, Broderick JP, Barsan WG, Sauerbeck LR, Zuccarello M, et al: The ABCs of measuring intracerebral hemorrhage volumes. Stroke 27:1304-1305, 1996

14. Li T, Wang D, Tian Y, Yu H, Wang Y, Quan W, et al: Effects of atorvastatin on the inflammation regulation and elimination of subdural hematoma in rats. J Neurol Sci 341:88-96, 2014

15. Lin LY, Huang CC, Chen JS, Wu TC, Leu HB, Huang PH, et al: Effects of pitavastatin versus atorvastatin on the peripheral endothelial progenitor cells and vascular endothelial growth factor in high-risk patients: a pilot prospective, double-blind, randomized study. Cardiovasc Diabetol 13:111, 2014

16. Liu L, Wei H, Chen F, Wang J, Dong JF, Zhang J: Endothelial progenitor cells correlate with clinical outcome of traumatic brain injury. Crit Care Med 39:1760-1765, 2011

17. Liu W, Bakker NA, Groen RJ: Chronic subdural hematoma: a systematic review and meta-analysis of surgical procedures. J Neurosurg 121:665-673, 2014

18. Markwalder TM, Steinsiepe KF, Rohner M, Reichenbach W, Markwalder H: The course of chronic subdural hematomas after burr-hole craniostomy and closed-system drainage. J Neurosurg 55:390-396, 1981

19. Miranda LB, Braxton E, Hobbs J, Quigley MR: Chronic subdural hematoma in the elderly: not a benign disease. J Neurosurg 114:72-76, 2011

20. Motiei-Langroudi R, Stippler M, Shi S, Adeeb N, Gupta $\mathrm{R}$, Griessenauer CJ, et al: Factors predicting reoperation of chronic subdural hematoma following primary surgical evacuation. J Neurosurg 129:1143-1150, 2018

21. Oikonomou E, Siasos G, Zaromitidou M, Hatzis G, Mourouzis $\mathrm{K}$, Chrysohoou C, et al: Atorvastatin treatment improves endothelial function through endothelial progenitor cells mobilization in ischemic heart failure patients. Atherosclerosis 238:159-164, 2015

22. Poulsen FR, Munthe S, Soe M, Halle B: Perindopril and residual chronic subdural hematoma volumes six weeks after burr hole surgery: a randomized trial. Clin Neurol Neurosurg 123:4-8, 2014

23. Prud'homme M, Mathieu F, Marcotte N, Cottin S: A pilot placebo controlled randomized trial of dexamethasone for chronic subdural hematoma. Can J Neurol Sci 43:284-290, 2016

24. Qiu S, Zhuo W, Sun C, Su Z, Yan A, Shen L: Effects of atorvastatin on chronic subdural hematoma: a systematic review. Medicine (Baltimore) 96:e7290, 2017

25. Quan W, Zhang Z, Tian Q, Wen X, Yu P, Wang D, et al: A rat model of chronic subdural hematoma: insight into mechanisms of revascularization and inflammation. Brain Res 1625:84-96, 2015

26. Rovlias A, Theodoropoulos S, Papoutsakis D: Chronic subdural hematoma: surgical management and outcome in 986 cases: a classification and regression tree approach. Surg Neurol Int 6:127, 2015

27. Soleman J, Nocera F, Mariani L: The conservative and pharmacological management of chronic subdural haematoma. Swiss Med Wkly 147:w14398, 2017

28. Song Y, Wang Z, Liu L, Wang D, Zhang J: The level of circu- 
lating endothelial progenitor cells may be associated with the occurrence and recurrence of chronic subdural hematoma.

Clinics (São Paulo) 68:1084-1088, 2013

29. Sun TF, Boet R, Poon WS: Non-surgical primary treatment of chronic subdural haematoma: preliminary results of using dexamethasone. Br J Neurosurg 19:327-333, 2005

30. Tang R, Shi J, Li X, Zou Y, Wang L, Chen Y, et al: Effects of atorvastatin on surgical treatments of chronic subdural hematoma. World Neurosurg 117:e425-e429, 2018

31. Thotakura AK, Marabathina NR: Nonsurgical treatment of chronic subdural hematoma with steroids. World Neurosurg 84:1968-1972, 2015

32. Wang B, Sun L, Tian Y, Li Z, Wei H, Wang D, et al: Effects of atorvastatin in the regulation of circulating EPCs and angiogenesis in traumatic brain injury in rats. J Neurol Sci 319:117-123, 2012

33. Wang D, Li T, Tian Y, Wang S, Jin C, Wei H, et al: Effects of atorvastatin on chronic subdural hematoma: a preliminary report from three medical centers. J Neurol Sci 336:237-242, 2014

34. Wang D, Li T, Wei H, Wang Y, Yang G, Tian Y, et al: Atorvastatin enhances angiogenesis to reduce subdural hematoma in a rat model. J Neurol Sci 362:91-99, 2016

35. Yao Z, Hu X, Ma L, You C: Dexamethasone for chronic subdural haematoma: a systematic review and meta-analysis. Acta Neurochir (Wien) 159:2037-2044, 2017

36. Zhang Y, Chen S, Xiao Y, Tang W: Effects of dexamethasone in the treatment of recurrent chronic subdural hematoma. World Neurosurg 105:115-121, 2017

\section{Disclosures}

The authors report no conflict of interest concerning the materials or methods used in this study or the findings specified in this paper.

\section{Author Contributions}

Conception and design: Jiang, D Wang, J Zhang. Acquisition of data: D Wang, Gao, Xu, Tian, Wei, Quan, Y Wang, Z Wang, Lei. Analysis and interpretation of data: Jiang, D Wang, Gao, Xu, Chen, Tian, Wei, Quan, Anderson, J Zhang. Drafting the article: Jiang, D Wang, Gao, Xu, S Zhang, Yue, Dong, J Zhang. Critically revising the article: Jiang, D Wang, S Zhang, Anderson, Dong, J Zhang. Reviewed submitted version of manuscript: Jiang, Gao, Dong. Statistical analysis: Chen, Anderson. Administrative/technical/material support: Yue, Lei. Study supervision: Jiang, Yue, J Zhang.

\section{Supplemental Information}

\section{Online-Only Content}

Supplemental material is available with the online version of the article.

Supplementary Tables and Figure. https://thejns.org/doi/suppl/ 10.3171/2019.11.JNS192020.

\section{Correspondence}

Rongcai Jiang: Tianjin Medical University General Hospital, Tianjin, China. jiang116216@163.com. 